2. To: (Recolving Organization)
R.J. Thomas/N1E10000/WAVS

5. Proj./Prog./Dapt./DN.:

Nuclear Material stabilization

8. Originator Remarke:

This EDT releases initial support analysis for the 236-z/291-z Sample Vacuum system cross-tie effort. The attached analysis evaluates pressure drop considerations for potential line sizes.

11. Recelver Remark: $\quad$ 11A. Design Basellne Document? $\square$ Yes $\square$ No

$\mathrm{N} / \mathrm{A}$
S.D. Ellingson/SF $840000 / \mathrm{MECH} / \mathrm{HVAC}$

6. Dacign Authority/Decion AgentCog. Engr.:

R. Thomas/S.Ellingson/R. Thomas
4. Rolated EDT No.:

N/A.

7. Purchaes Order No.:

$N / A$

9. Equip./Componont No.:

$\mathrm{N} / \mathrm{A}$

10. Syatom/Bidg./Faciliny:

23A/234-5Z/PFP

12. Major Alem. Dwg. No.:

N/A

13. Permit/Permk Application No.:

$\mathrm{N} / \mathrm{A}$

14. Required Response Dats:

N/A

DATA TRANSMITTED

15.

\begin{tabular}{|l|l|l|}
\hline $\begin{array}{c}\text { (C) Sheet } \\
\text { No. }\end{array}$ & (D) Rov. \\
No. & (E) Tile or Desecription of Data Tranamitted
\end{tabular}

(A)

(B) Document/Drawing No.
(F)

Pressure Drop Analysis

(G)

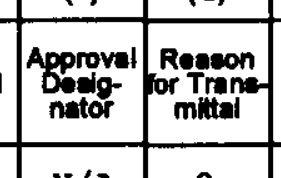

(H)

(l)

O Pressure Drop Analysis

\begin{tabular}{|l|l}
\hline 1 & HNF-6328 \\
\hline & \\
\hline & \\
\hline & \\
\hline & \\
\hline & \\
\hline & \\
\hline
\end{tabular}

16.

\begin{tabular}{|c|c|}
\hline Approval Designator (F) & \\
\hline $\begin{array}{c}\text { E, S, Q D OR N/A } \\
\text { (See WHC-CM-3-5, } \\
\text { Sec. 12.7) }\end{array}$ & $\begin{array}{l}\text { 1. Approval } \\
\text { 2. Rolease } \\
\text { 3. Information }\end{array}$ \\
\hline
\end{tabular}

17.

\begin{tabular}{|c|c|c|}
\hline $\begin{array}{l}\text { (G) } \\
\text { Rea- } \\
\text { son }\end{array}$ & $\begin{array}{c}(\mathrm{H}) \\
\text { Disep. }\end{array}$ & (L) Date (M) MSIN \\
\hline 2 & 1 & Design Authority $K \sqrt{1 \text { hemenos } \$ 1160}$ T4.20 \\
\hline 2 & 1 & Dockign Agent \\
\hline 2 & 1 & 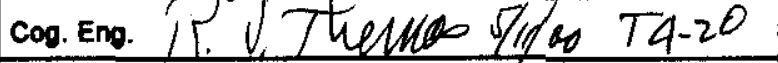 \\
\hline 2 & 1 & 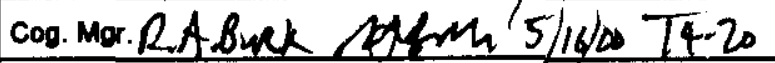 \\
\hline & & QA \\
\hline & & Safoty \\
\hline & & Env. \\
\hline
\end{tabular}

18.

S. Finen Signature of EDT Orginator
19.

$\frac{5 / 5 / 60}{\text { Date }}$

Date

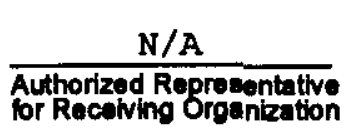

Reason for Tranamittal (G)

4. Review

8. Diat. (Recelpt Acknow. Required)

KEY
20.

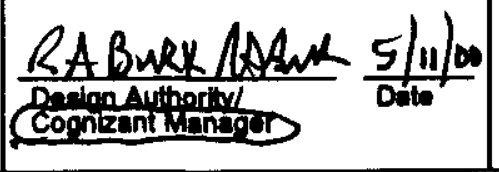

Diepockion (H) \& (I)

1. Approved

2. Approved w/comment

3. OLispproved w/comment

4. Reviowed no/comment 5. Reviowed w/comment 6. Recoipt acknowiedged SIGNATURE/DISTRIBUTION

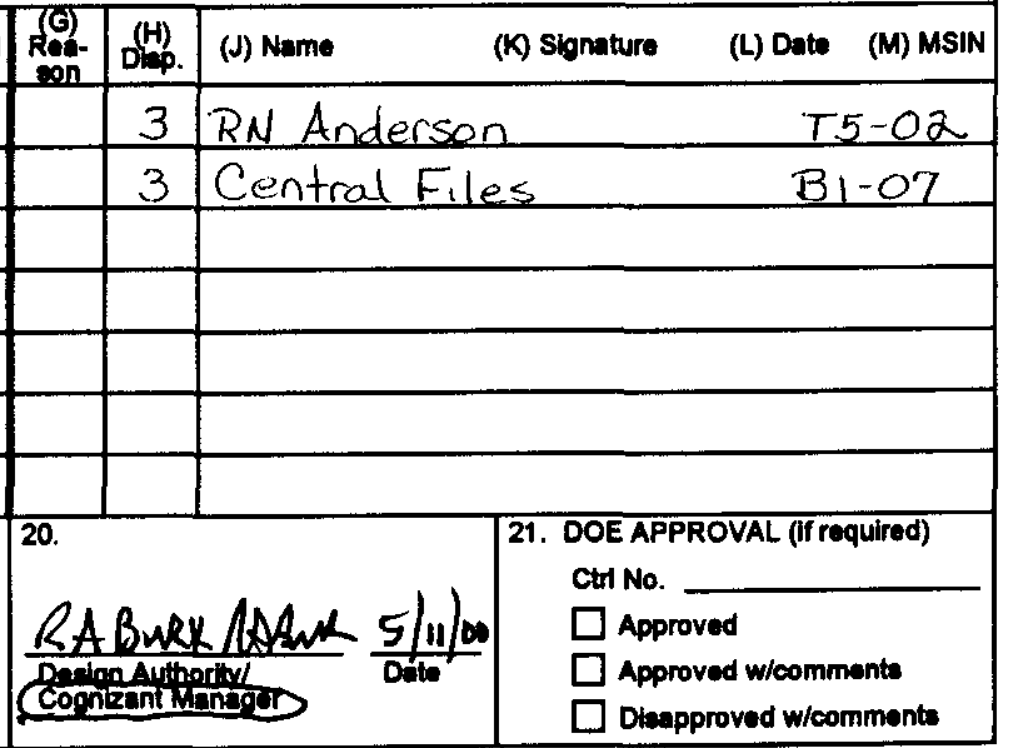


HNF-6328

Revision 0

\section{6-Z/291-Z Vacuum Pump System Pressure Drop Analysis For Line Size Selection}

Prepared for the U.S. Department of Energy Assistant Secretary for Environmental Management

Project Hanford Management Contractor for the

U.S. Department of Enorgy under Contract DE-ACOQ-86RL13200

Fluor Hanford

P.O. Box 1000

Richland, Washington 


\section{6-Z/291-Z Vacuum Pump System Pressure Drop Analysis For Line Size Selection}

SD Ellingson

Fluor Hanford

Date Published

May 2000

Prepared for the U.S. Department of Energy

Assistant Secretary for Environmental Management

Project Hanford Management Contractor for the

U.S. Department of Energy under Contract DE-AC08-86RL13200

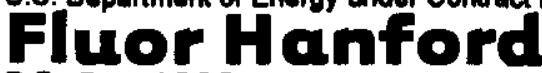

P.O. Box 1000

Richland, Washington
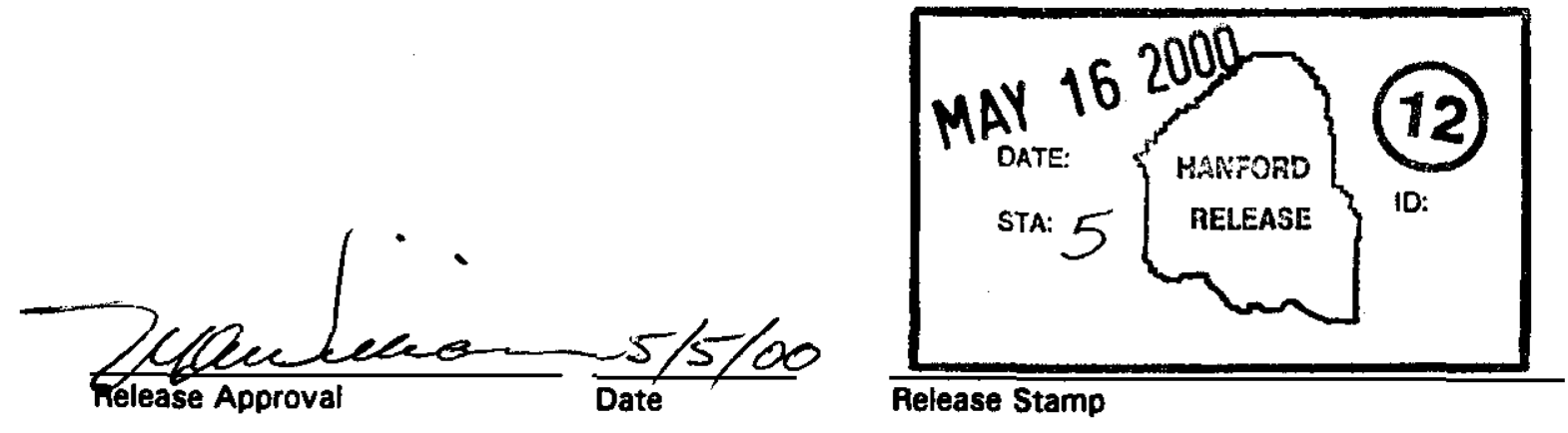
HNF-6328

Revision 0

LEGAL DISCLAIMER

This report wes prepared as an eccount of work sponaored by an agency of the United States Government. Noither the Uniked States Government nor any agency thereof, nor any of their employees, nor any of their contractors, subcontractors or their employees, makes any warranty, expreses or impled, or aseumes any legal liability or responelbiliky for the sccuracy. complateneses, or any third party's uas or the results of such ues of any information, apparatus, product, or proceas diacloeed, or represents that lis uas would not infiringe privately owned righte. Reference hervin to any epectilc commercial product, procese, or service by trade name, trademark, manufacturer, or otherwase, does not necesearily constitute or Imply its endoreement, recommendation, or favoring by the United States Government or any agency thereof or the contractore or subcontractors. The views and opinions of authors expresesed herein do not necesearlly state or reflect those of the United States Government or any agency thereof.

This report has been reproduced from the best avallable copy.

Tokal Pagea: 
HNF-632B, REVO, PS 1

\section{Fluor Federal Services}

Status and description of the attached Calculation Sheets.

Discipline: Mechanical

Project No. \& Title: Plutonium Finishing Plant Contract 5204, Release 22

Calculations: 236-Z/291-Z Vacuum Pump System Pressure Drop Analysis

These calculations apply to:

Drawing No.

Rev. No.

Drawing No.

Rev. No.

Other (Study, CDR)

Rev. No.

The status of these calculations is:

\ Final Calculations

$\square$ Void Calculations (reason voided):

Were calculations incorporated into the final drawings?

$\square \quad$ Yes

$\square \quad$ No

Were calculations verified by independent "check" calculations?

प Yes

$\square \quad$ No

Original and Revised Calculation Approvals

\begin{tabular}{l|c|c|c} 
& $\begin{array}{c}\text { Rev. } 0 \\
\text { Signature/Date }\end{array}$ & $\begin{array}{c}\text { Rev. 1 } \\
\text { Signature/Date }\end{array}$ & 2 \\
\hline Originator & Signature/Date
\end{tabular}

\section{INDEX}

Calculation Sheet Page No.

Description

1

2 thru 10

Al thru $\mathrm{A4}$
Analysis "Given/Required/Solution"

Pressure Drop Calculations

Appendix 
HNF-6328, REV 0, P9 2

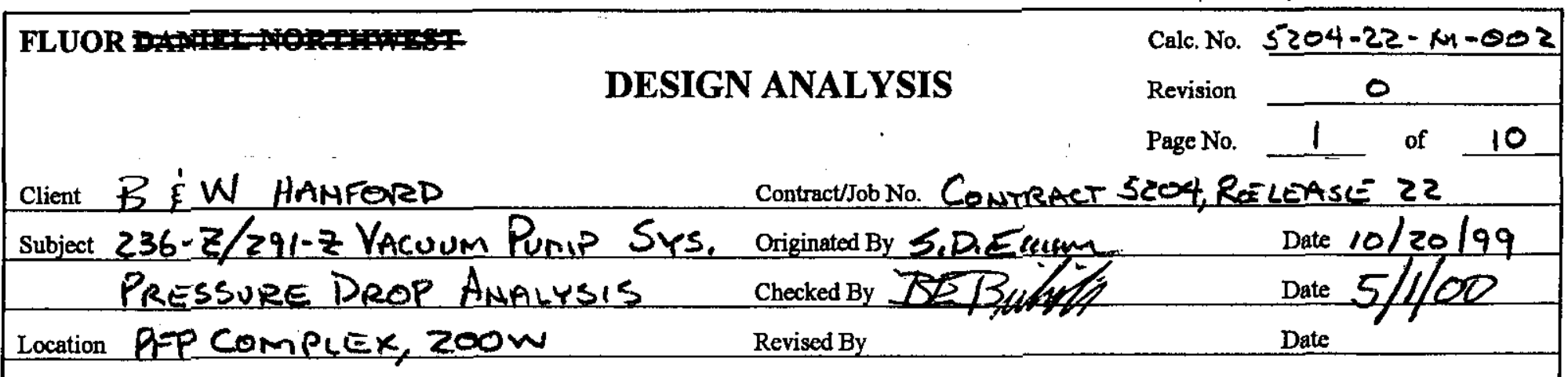

GIVEN THERE ARE CURRENTLY TWO SAMPLE VACUUM PUMP SYSTEMS AT THE PF FACILITY FOR OBTAINING RECORd SAMPLE'S AND AIR MOMITORING, OME PUMP SYSTEM, THE RF SYSTEM, IS LOCATED IN $236-Z$ AND SERVICES ONLY $236-Z$. THE SECOND SYSTEM, THE PIP SYSTEM, IS LOCATED IN 291-Z AND SERVICES THE Z91-Z, 232-Z, 2736-Z AND 234-5Z SYSTEMS. IT HAS BEEN REQUESTED TO COMBINE THE PREF SYSTEM WITH THE PEP SYSTEM AND ELIMINATE THE RF VACUUM PUMPS. FOUR PIPING options to achieve this goal haVe be determined. SEE apPendiX SHEETS AI $\rightarrow$ AS.

SYSTEM FLOW REQUIREMENTS FOR THE PR SYSTEM HAS BEEN DETERMINED TO BE 196 CAM AT 17 IN HI VACUUM. REF HNF-6327.

FOR PIPING DRAWINGS, SEE THE FOLLOWING:

$H-2-29645,29648,29651,29657,29658$ AND 29659 .

REQUIRED DETERMINE THE INHERENT PRESSURE DROP OF EACH NEW SYSTEM OPTION. DETERMINE PRESSURE DROP FOR OTHER LINE SIZES TO ASSIST IN FINAL LINE SIZE SELECTION.

SOLUTION THE EQuATIONS AND TABLES IN CRANE Flow OF FlUIDS, TECHNICAL PAPER No, $410,19^{\text {TM }}$ PrINTING, 1980 EDITION, WILL BE USED TO SOLVE THIS PROBLEM. UNLESS OTHERWISE NOTED, ALL REFERENCES MADE WITHIN THIS ANALYSIS ARE WITH RESPECT TO THIS PUBLICATION. THE BASIC DARCY FORMULA TO BE USED: $\triangle P=\frac{3.62 \mathrm{KPq}}{\mathrm{d}^{4}}$

SYSTEM PARAMETERS: REFERENCES:

CRANE EQ 3-14

FLUID = AIR Q $68^{\circ} \mathrm{F}$ AMD 17 INtI VACUUM

$q=$ FLOWRATE $=196 \mathrm{CFM}=3.267 \mathrm{FT} / \mathrm{sEC}$

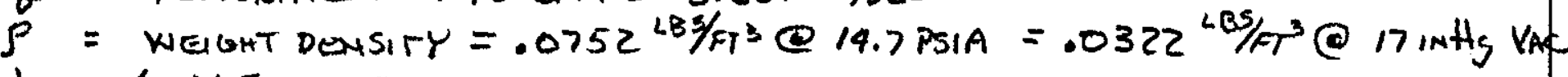

$d_{6}=6.065 \mathrm{~N} ?$

$\left.\begin{array}{l}d_{4}=4.026 \mathrm{iN} \\ d_{2}=2.067 \mathrm{~N}\end{array}\right\}$ INTERNAL PIPE DIAMETERS

E-NW-123B (5/98) 
HNF-6328, Rev 0, P9 3

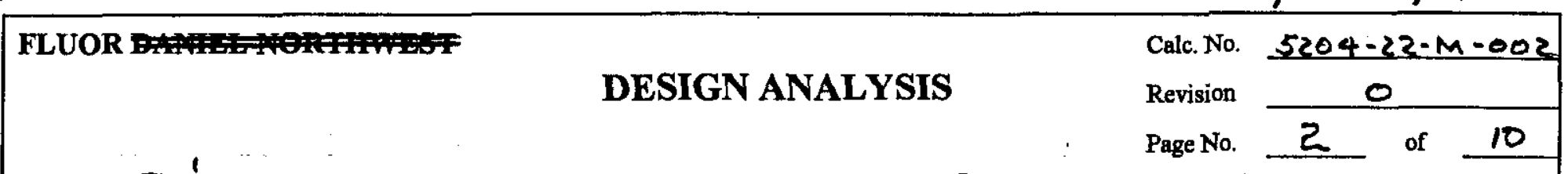

Client B\&W HANFORD

Subject $236-z / 291-z$ VAcuuM Pump SYS. Originated By SiD.Eucum

pressure Drop Analysis Checked By BePidifi

Location PFP COMIRLEX, $200 \mathrm{~W}$

Revised By

$10 / 20 / 99$

FOR OPTIOM \#1

Date $5 / 1 / 60$

Date

THE INTENT OF OPTION \#I IS TO USE ONE OR MORE EXISTING $Z$ " PIPELINES RUNNING BETWEEN BUILDINGS $236-Z$ AND $291-Z$. TO SIMPLIFY CALCULATIONS, EVALUATE THE NEW PIPING FROM THE EXISTING PIPING SEPARATELY AND CONBIDCRE $2 ", 4 "$ ? 6 "PIPE SIZE OPTIONS. Also ASSUME THE HERA FILTER $\triangle P$ IS CONSTANT AMD ADD SEPERATIELY.

TOTAL $\triangle P=\Delta P($ NEW PIPING $)+\Delta P($ EXISTING PIPING $)+\Delta P$ FILTER

- For NEW 6" PIPING, determine pK AND $\triangle P$. From DRAWING Revient COMPONENTS INCLUDE 52 AT OF PIPE AND (8) $90^{\circ}$ elBows: Note, Assume new Piping is same As Existing, Ie, PiPe Code M7, 1-2-31750

$$
\begin{aligned}
& K(P, P E)=\frac{f L}{D} \text { CRANE 3-15, ASSUME TURBULENT Flow } f \text { 'f }=.015 \text { CrRAME } \\
& K(P 1 P E)=(.015) \frac{(52)(12)}{6.065}=1.543
\end{aligned}
$$

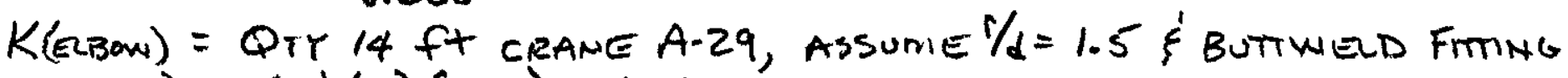

$$
\begin{aligned}
& K(\text { EcON })=(8)(14)(.015)=1.68 \\
& \Sigma K=1.543+1.68=3.223 \\
& \Delta P_{6}=3.62 \frac{\mathrm{kK \rho q^{2 }}}{d^{4}}=\frac{(3.62)(3.223)(.0322)(3.267)^{2}}{(6.065)^{4}}=.00296 \mathrm{ps} 1=0.00603 \mathrm{in} \mathrm{H}^{4}
\end{aligned}
$$

- For new 4" PIPING, SAME flow AMd fittings only difFerent SIZE. FIND $\angle K$ AND $\triangle P$ :

$$
\begin{aligned}
& K(P, P E)=\frac{f L}{D}=\frac{(.017)(52)(12)}{4.026}=2.634 \quad \sum \sum K=2.634+1.904=4.538 \\
& K(\text { ELBOW })=0.414 \mathrm{ft}=(8)(14)(.017)=1.904
\end{aligned}
$$

E-NW-123B (5/98) 


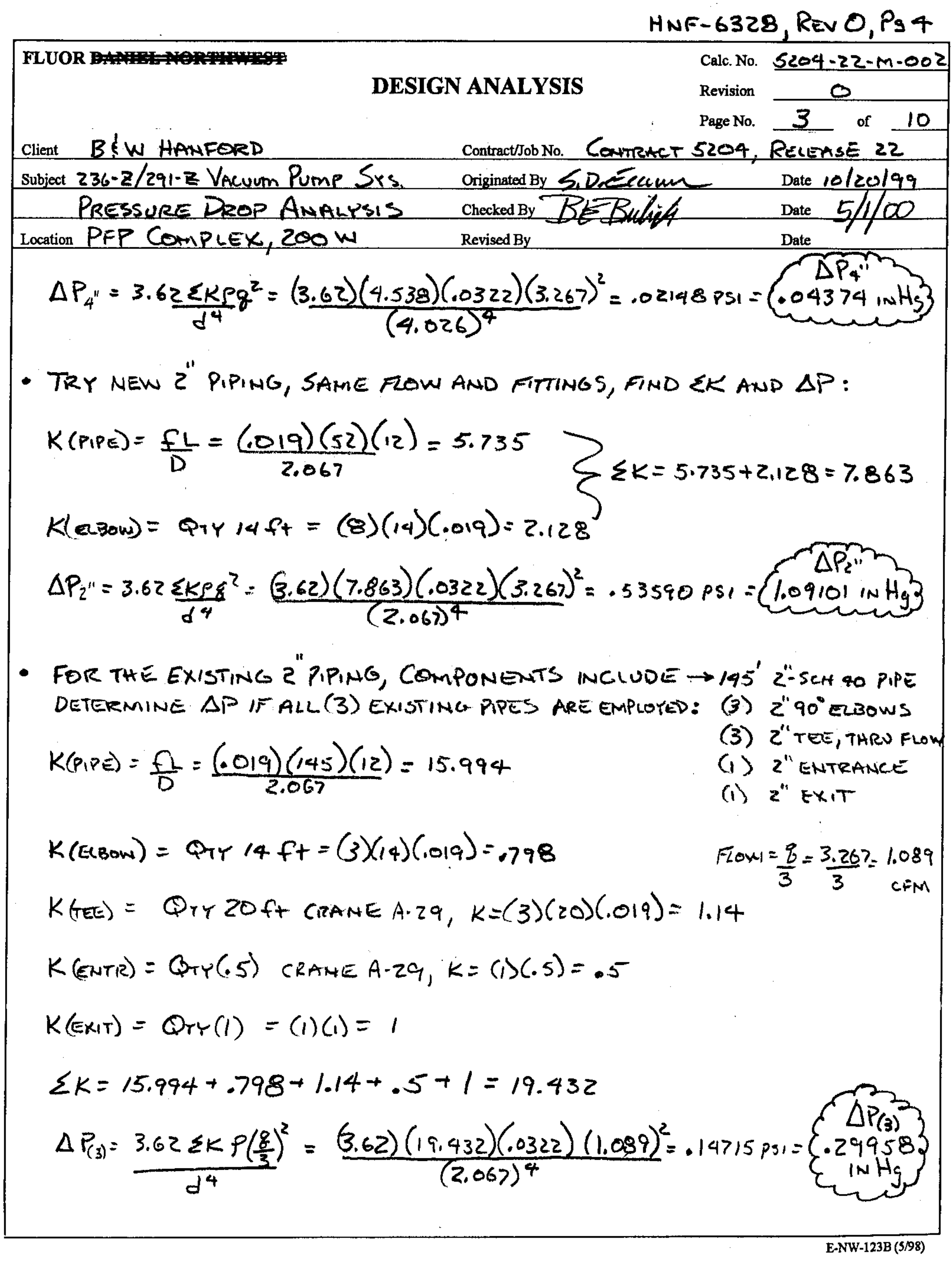


HNF- 6328, REv O, PS 5

Call. No. $5204 \cdot 22-M-002$

DESIGN ANALYSIS

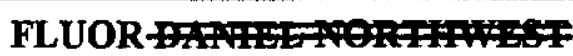

Revision

Page No. 4 of 10

Client BE'W HANFORD ContractJob No. CONTRACT 5204 , RELEASE 22 Subject $236-z / 291-z$ VACuUm PUMP SYS Originated By S.D. Eccum Date $10 / 20199$ Pressure prop Analysis Date $5 / 4 / 0$ Location PFP COMPLEX, $200 \mathrm{~W}$ Checked By BE Bulfift Date

- determine sp if only (2) of the (3) existing pipes are employed:

COMPONENTS AND ER AS BEFORE, FLOW $=\frac{q}{2}=\frac{3.267}{2}=1.6335$

$$
\Delta P(2)=(\Delta P(3))\left(\frac{q(2)}{q(3)}\right)^{2}=(.29958)\left(\frac{1.6335}{1.089}\right)^{2}=\underbrace{.67405 \text { in Hg }}_{\Delta P(2)}
$$

- Determine $\triangle P$ if only (i) of the (3) existing pipes are employed:

$$
\begin{aligned}
& \text { AS BEFORE WITH FLOW }=\frac{q_{1}}{1}=q_{1} \\
& \Delta P(1)=(\Delta P(3))\left(\frac{q(1)}{q(3)}\right)^{2}=(.29958)\left(\frac{3.267}{1.089}\right)^{2}=\underbrace{2.69622} \mathrm{inHg}
\end{aligned}
$$

- for hera filter pressure drop, assume constant ce $4 \mathrm{inh}$. O:

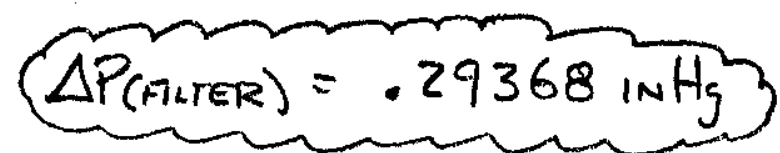

Option \#1 Summary:

* if new piping is 6", inherent $\triangle P=.00603 \mathrm{inHg}$ IF NEW PIPING is 4", INITERONT $\triangle P=.04374 \mathrm{inHg}$

A IF NEU PIPING is 2 ; INHERENT $\triangle P=1.09101 \mathrm{iNHg}$

* if (3) existing 2" LINES Are employed, inHerent $\triangle P=.29958 \mathrm{inH}$ IF (2) EXISTING Z" LINES ARE EMPLOYQND, INHERENT $\triangle P=.67405 \mathrm{inH}$ A IF CI) EXISTINGZ"LINE 13 EMPLOYED, INHERENT $\triangle P=2.69622 \mathrm{inH}$

FILTER $\triangle P=.29368 \mathrm{iNHg}$

* BEST CASE TOTAL $=.00603+.29958+.29368=.59929 \mathrm{inttg}$

\# WORST CASE TOTAL $=1.09101+2.69622+.29368=4.08091 \mathrm{in} \mathrm{Hg}$

E-NW-123B (5/98) 
MNF- 6328, REv 0, P9 6

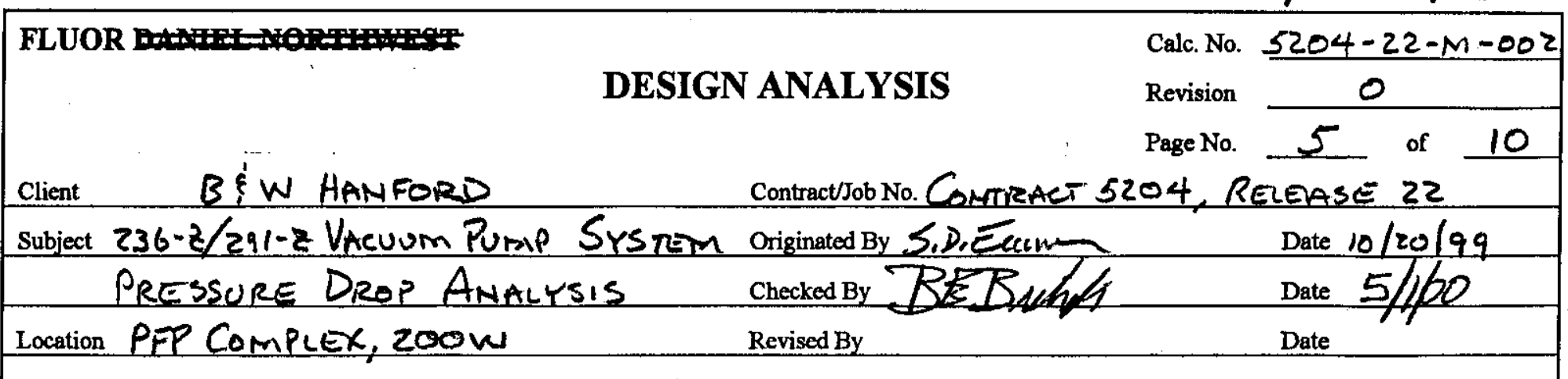

OPTION \#2

Option 2 employed all new pipe And fittings. Determine $Z K$ and $\triangle P$

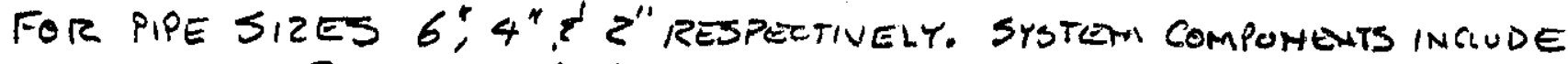
297 FT OF PIPE AND (12) $90^{\circ}$ ELBOW NS.

- FOR 6" PIPE:

$$
\begin{aligned}
& \begin{array}{l}
K(\text { PIPE })=\frac{f L}{D}=\frac{(.015)(297)(12)}{6.065}=8.814 \quad\left\{\sum K=8.814+2.52=11.334\right. \\
K(\text { ELBOW })=Q_{\text {TY }} 14 \mathrm{ft}=(12)(14)(.015)=2.52
\end{array} \\
& \Delta P_{G^{\prime \prime}}=\frac{3.62 \sum K \rho q^{2}}{d^{4}}=\frac{(3.62)(11.334)(.0322)(3.267)^{2}}{(6.065)^{4}}=.01042 P_{51}=\underbrace{.02121{ }^{\prime \prime N H} H_{3}}
\end{aligned}
$$

- For 4" PIPE:

$$
\begin{aligned}
& K(P, P E)=\frac{f L}{D}=\frac{(.017)(297)(12)}{4.026}=15.049 \\
& K(\text { ELBOW })=\text { QTY } 14 f=(12)(14)(.017)=2.856 \\
& \Delta P_{4^{\prime \prime}}=\frac{3.62 \sum K \rho q^{2}}{d^{4}}=\frac{(3.62)(17.095)(.0322)(3.267)^{2}}{(4.026)^{4}}=.08478 P_{S 1}=17.049+2.856
\end{aligned}
$$

- for Z" pIPE:

$$
\begin{aligned}
& K(p, 1 E)=\frac{G L}{D}=\frac{(.019)(297)(12)}{2.067}=32.760 \\
& K(\text { ELBOW })=\text { QTY 14f } f=(12)(19)(.019)=3.192
\end{aligned} \begin{aligned}
& \sum K=32.060+3.192 \\
& =35.952
\end{aligned}
$$

E-NW-123B ( $(5 / 98)$ 
HNF-6328, REV O, PS 7

DESIGN ANALYSIS

Call. No. $5204-22-M-002$

Page No.

Client BI'W HANFORD Subject 236-z/291-z VAcuUm Pump SYS. ContractJob No. Contract 5204, RElents 22 Pressure Drop AnAlysis Originated By S, D. Flew Date $10 / 20 / 90$ Location PFP COMPLEX, zoom Revised By Date $5 / / 20$

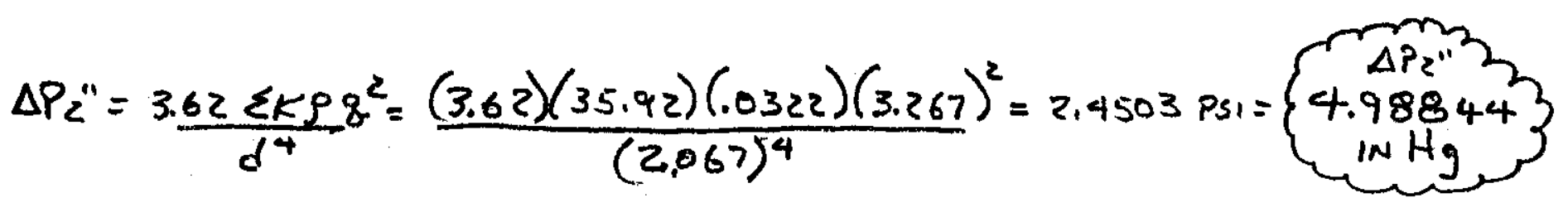

OPTION 2 SumMary

* if new piping is 6", inherent $\Delta P=.02121 \mathrm{inH}$

IF NEW PIPING IS 4", INHERENT $\triangle P=.17261 \mathrm{iNH}^{\circ}$

A IFNEN, PIPING is $2 "$, INHERENT $\triangle P=4.98844$ in $\mathrm{Hg}_{g}$

FILTER $\triangle P=.29368 \mathrm{in} \mathrm{Hg}$ (AS BEFORE)

* best case total $=.02121+.29368=.31489 \mathrm{~N} \mathrm{Hg}$

$\triangle$ Worst case total $=4.98844+.29368=5.28212 \mathrm{in} \mathrm{Hg}$

Option \#3

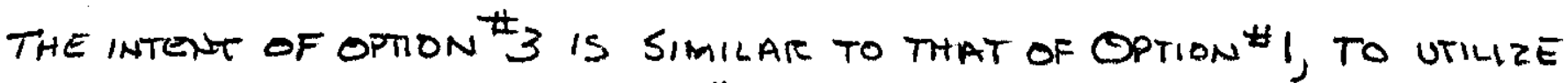
ONE OR ALL OF THE EXISTING Z" PIPELINES BETWEEN Z36-Z AND Z91-Z, THIS CALCULATION IS SIMILAR TO OPTION \# I WITH THE EXCEPTION THAT THE FOLLOWING NEW PIPE AND FITTINGS ARE EMPLOYED $\rightarrow 136 \mathrm{FT}$ PIPE FIND NEW ER AND $\triangle P$ FOR VARIOUS PIPE SIZES:

(9) $90^{\circ}$ ElBows

(2) $45^{\circ}$ EB Bows

- FOR NEW 6" PIPING:

(1) TEE, BRANCH

$$
\begin{array}{ll}
K(\text { PIPE })=\frac{f L}{D}=\frac{(.015)(136)(12)}{6.065}=4.036 & K(\text { TEE })=60 f=(60)(.015)=.9 \\
K\left(10^{\circ}\right)=\operatorname{DTY} 14 f=(9)(14)(.015)=1.89 & \left\{\begin{array}{l}
\sum K=4.036+1.897 .45+.9 \\
=7.276
\end{array}\right. \\
K\left(45^{\circ}\right)=\operatorname{QTY} 15 f=(2)(15)(.015)=.45
\end{array}
$$

(ASSUMED MITRE BEND)

E-NW-123B (5/98) 
HNF-6328, REV O, PS 8

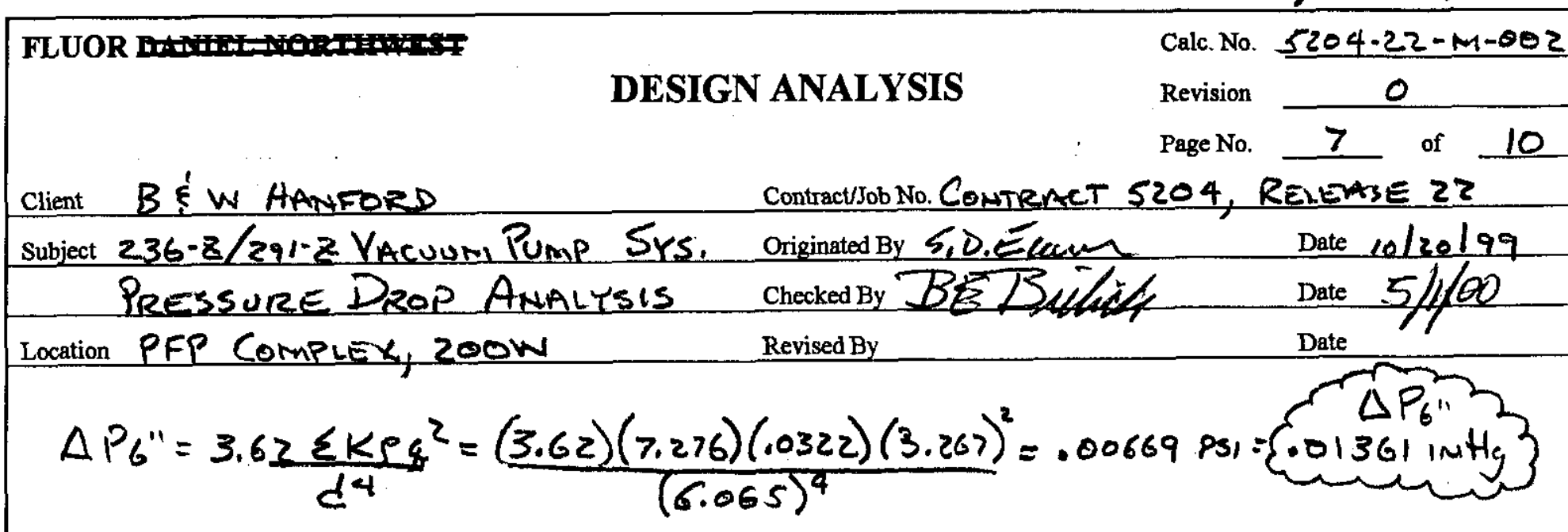

- For nen 4"Piping:

$$
\begin{aligned}
& \left.K(P, P E)=\frac{f L}{D}=\frac{(.017)(136)(12)}{4.026}=6.890\right) \\
& \left.K\left(90^{\circ}\right)=\phi_{1 Y} 14 f=(9)(14)(.017)=2.142\right\} \sum K=6.890+2.142+.51+1.02 \\
& K\left(45^{\circ}\right)=\operatorname{Qry} 15 f=(2)(15)(.017)=.51 \\
& =10.562 \\
& K(\text { TEE })=60 f=(60)(.017)=1.02
\end{aligned}
$$

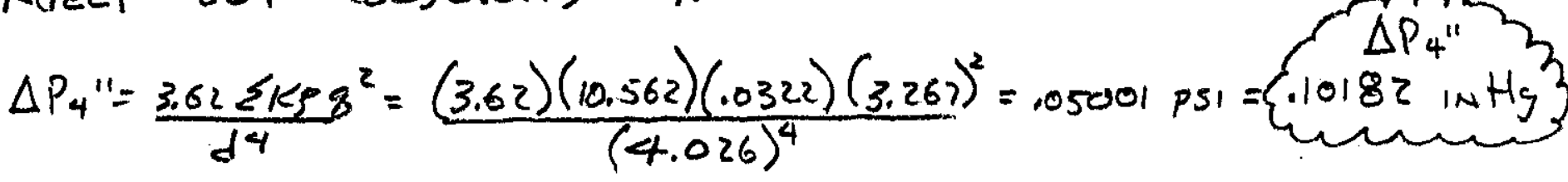

- for nen 2 " piping:

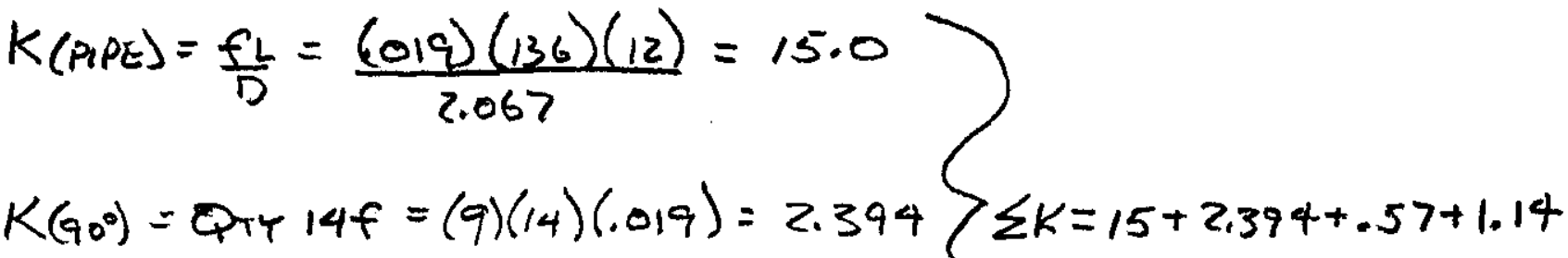

$$
\begin{aligned}
& K\left(45^{\circ}\right)=\operatorname{Qrr} 15 f=(2)(15)(.019)=.57 \\
& K(\text { TEE })=60 f=(60)(.019)=1.14 \\
& =19.104 \\
& \Delta P_{2}^{\prime \prime}=\frac{3.62 \sum K P q^{2}}{d^{9}}=\frac{(3.62)(19.109)(.0322)(3.267)^{2}}{(2.067)^{4}}=1.30204 P_{51}=2.65072 \mathrm{inH}^{3}
\end{aligned}
$$

E-NW-123B (5/98) 
HNF-6328, REV O, PS 9

DESIGN ANALYSIS

Call. No. $5204 \cdot 22-M-\infty 02$

FLUOR

Revision $\frac{0}{\mathscr{8} \text { of } 10}$

Client BE'W HANFORD Contract/Job No. ConTraCt 5204, RELASE 22 Subject $236-z / 291-z$ VACuUM PUMP SYS, Originated By $5, D, E$ eam Date $10 / 20 / 99$ Pressure Drop Analysis Checked By Be Butut y Date $5 / 4 / 100$ Location PFP COMPLEX, zoON Revised By

Date

- For existing z" pipelines, components and flow do not change to that analyzed in option \#1, $\therefore$ All combinations same as option "l.

OPTION \# 3 SUMMARY

* if new piping is 6", inHerent $\triangle P=.01361 \mathrm{inH}$ IF NEW PIPING IS 4," INHERENT $\triangle P=.10182 \mathrm{inHg}$

\# IF NEW PIPING is 2 ; INHERENT $\triangle P=2.65072 \mathrm{NH}$

* if (3) Existing Z" LINES ARE EMPLOYED, INHERENT $\triangle P=.29958 \mathrm{inHg}$ IF (2) EXISTING $2 "$ "LIES ARE EMPLOYED, INHERENT $\triangle P=.67405 \mathrm{iNH}$

\# IF (i) EXISTING Z"LHE IS EMPLOYED, INHERENT $\triangle P=2.69622$ iN HG FILTER $\triangle P=.29368 \mathrm{inHS}$ (AS BEFORE)

* Best case total $=.01361+.29958+.29368=.60687 \mathrm{in} \mathrm{Hg}$

A worst case toTAL $=2.65072+2.69622+.29368=5.64062 \mathrm{inttg}$

OPTION ${ }^{\#}$

LIKE OPTION Z, OPTION 4 EMPLOYS ALL NEW PIPE AMD FITTINGS. DETERMINE NEW pK AND $\triangle P$ FOR PIPE SIZES 6,"4", $2 "$, NEW COMPONENTS INCLUDE: 289 FT PIPE, (12) $90^{\circ}$ elBOWS, (2) $95^{\circ}$ ERBOWS '́(1) TEE

- For new 6" piping:

$$
\begin{aligned}
& K(P, P E)=\frac{f L}{D}=\frac{(.015)(289)(12)}{6.065}=8.577 \quad K(\tau \in t)=60 f=(60)(.015)=.9 \\
& K\left(90^{\circ}\right)=Q_{T Y} 14 f=(12)(14)(.015)=2.52 \\
& K(4)^{\circ}=Q_{T Y 15 f}=(2)(15)(015)=.45
\end{aligned} \quad \begin{gathered}
\sum K=8.577+2.527 .45+.9 \\
=12.447
\end{gathered}
$$

E-NW-123B (5/98) 
HNF-6328, REVO, PS 10

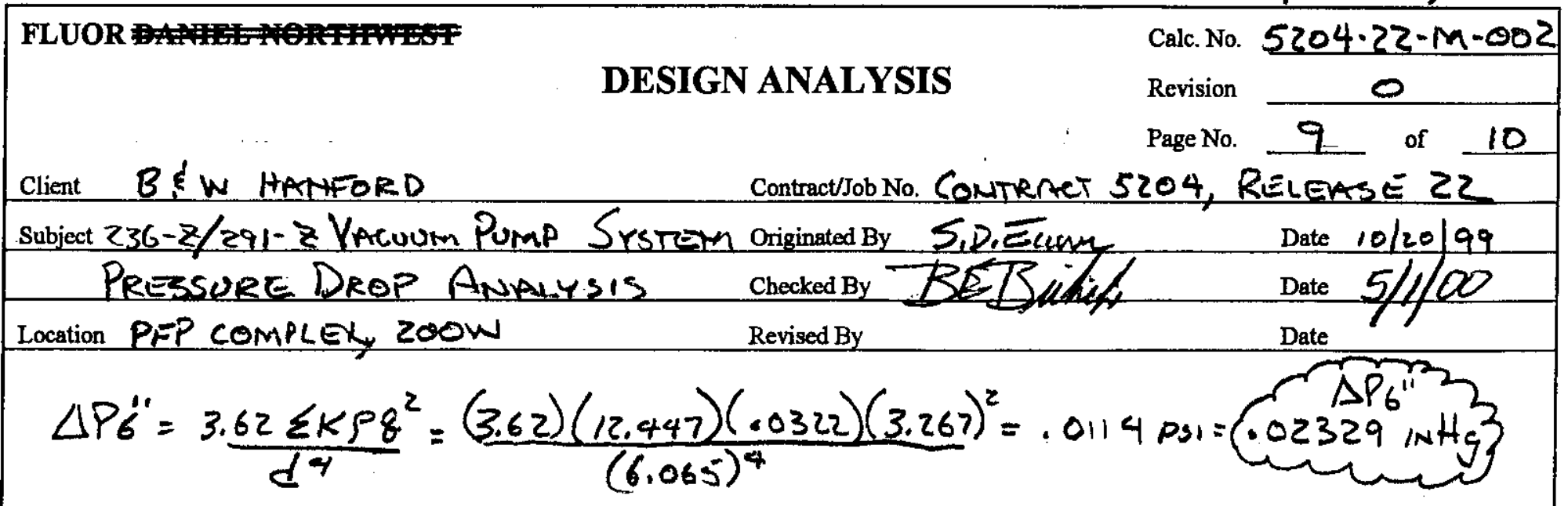

- for nen 4"piping:

$$
\begin{aligned}
& K(P, P C)=\frac{f_{L}}{D}=\frac{(.017)(289)(12)}{4.026}=14.643 \\
& \left.K\left(90^{\circ}\right)=Q_{\text {Ty }} 14 f=(12)(14)(.017)=2.856\right\} \begin{aligned}
\sum K & =14.643+2.856+.51+1.02 \\
& =19.029
\end{aligned} \\
& K\left(45^{\circ}\right)=\operatorname{Qr} 415 f=(2)(15)(.017)=.51 \\
& K(\text { Tet })=60 f=(60)(.017)=1.02 \\
& \Delta P_{4}^{\prime \prime}=\frac{3.62 \sum K \rho q^{2}}{d^{4}}=\frac{(3.62)(19.029)(.0322)(3.267)^{2}}{(4.026)^{4}}=.0901 P_{11}=.18345 \mathrm{inHg}^{3}
\end{aligned}
$$

- for new z" piping:

$$
\begin{aligned}
& \begin{array}{l}
K(P, P E)=\frac{f L}{D}=\frac{(.019)(289)(12)}{2.067}=31.878 \\
K(90)=\operatorname{QrY} 14 f=(12)(14)(.019)=3.192 \quad S \angle K=31.878+3.192+.57+1.14
\end{array} \\
& K\left(45^{\circ}\right)=Q_{\text {TY } 15 f}-(2)(15)(.019)=.57 \\
& =36.78 \\
& K(\text { TEE })=60 f=(60)(.019)= \\
& 1.14
\end{aligned}
$$

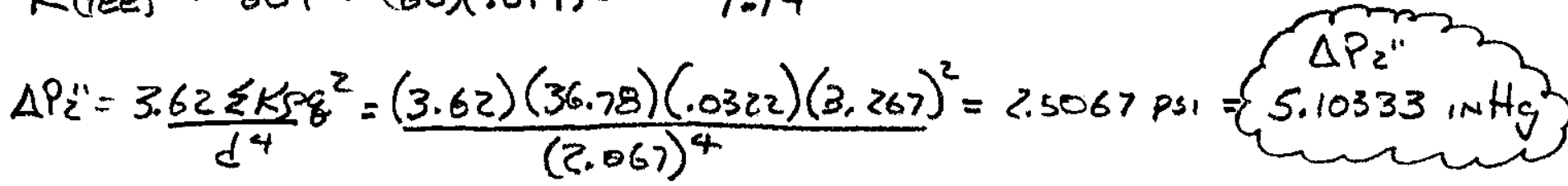

E-NW-123B (5/98) 
HNF-6328, REV O, PS 11

DESIGN ANALYSIS

Call. No. 5204-22-M-002

Revision 0

Page No. 10 of 10

Client BE W HANFORD

ContractJob No. Contract SZO4, RELETSE 22

Subject $236-2 / 291-2$ VACuUM PumP SYSTEM Originated By S.D. E fam

Date 10/20199

pressure prop Analysis Checked By BSBulyty

Date $5 / 1 / 00$

Location PFP COMPLEX, 200 W

Revised By

Date

OPTION * 4 SUMMARY

* IF NEW PIPING IS 6", INHERENT $\triangle P=.02329 \mathrm{inH}$

IF NEW PIPING is \&, INHERENT $\triangle P=.18345 \mathrm{inH}$

\# IF NEW PIPING IS $2 "$ ", INHERENT $\triangle P=5.10333 \mathrm{im} \mathrm{Hg}$

FILTER $\triangle P=.29368 \mathrm{inHS}$ (AS BEFORE)

* BEST CASE TOTAL $=.02329+.29368=.31697 \mathrm{inHg}$

A WORST CASE TOTAL $=5.10333+.29368=5.39701 \mathrm{imHg}$

CHECK COMPRESSIBILITY:

THE ABOVE CALCULATIONS DID NOT CHECK FOR THE NEED TO CORRECT For expansion FACTOR (REF Crane for COMPRESSIBLE FLOW). ALL CONCLUSIONS MUST BE CORRECTED WITH EXPANSION FACTOR "Y" IF $\triangle P>10 \% P_{1} \ldots$ CHECK TO SEE IF THO IS REQUIRED:

$$
\begin{aligned}
& P_{1}=17 \mathrm{inHg} \text { VACUUM }=6.3 \mathrm{PSIA} \quad \therefore 10 \% P_{1} \Rightarrow .63 \mathrm{PSIA}=1.28257 \mathrm{inHg} \\
& \text { OPTION * best case total }=.59929 \mathrm{inH} \text {. } \therefore 1.2825\rangle \text { OK } \\
& \text { WORSTCASE TOTAL }=4.08091 \mathrm{inHG} \therefore>1.28257 \mathrm{NO} \text { ! }
\end{aligned}
$$

BY OBSERVATION OF OTHER TOTALS, IT IS REALLY SEEN THAT THE WORST CASE CONDITIONS FOR EACH OPTION WOULD BE GREATER THAN $10 \%$ OF PI AND WOULD REQUIRE CORRECTION. THIS MIL BE ACCOUNTED FOR IN ADDITIONAL ANALYSIS IF THE CUSTOMER CONSIDERS SUCH ANE OPTION.

CONCLUSION SEE EACH OPTION SUMMARY FOR APPLICABLE $\triangle P$ S. USE THIS INFORMATION FOR FINAL PIPE SELECTION.

E-NW-123B (5/98) 


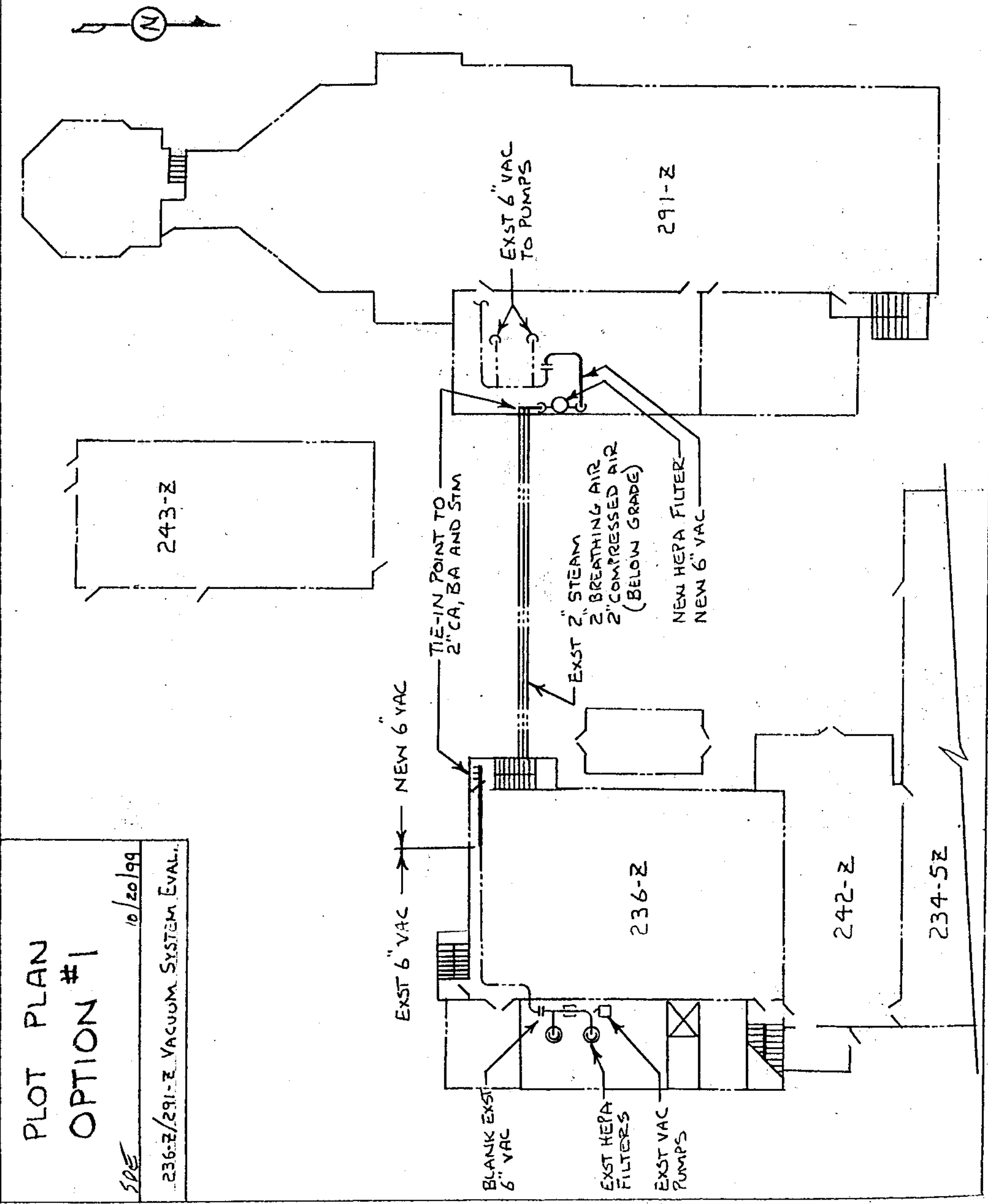




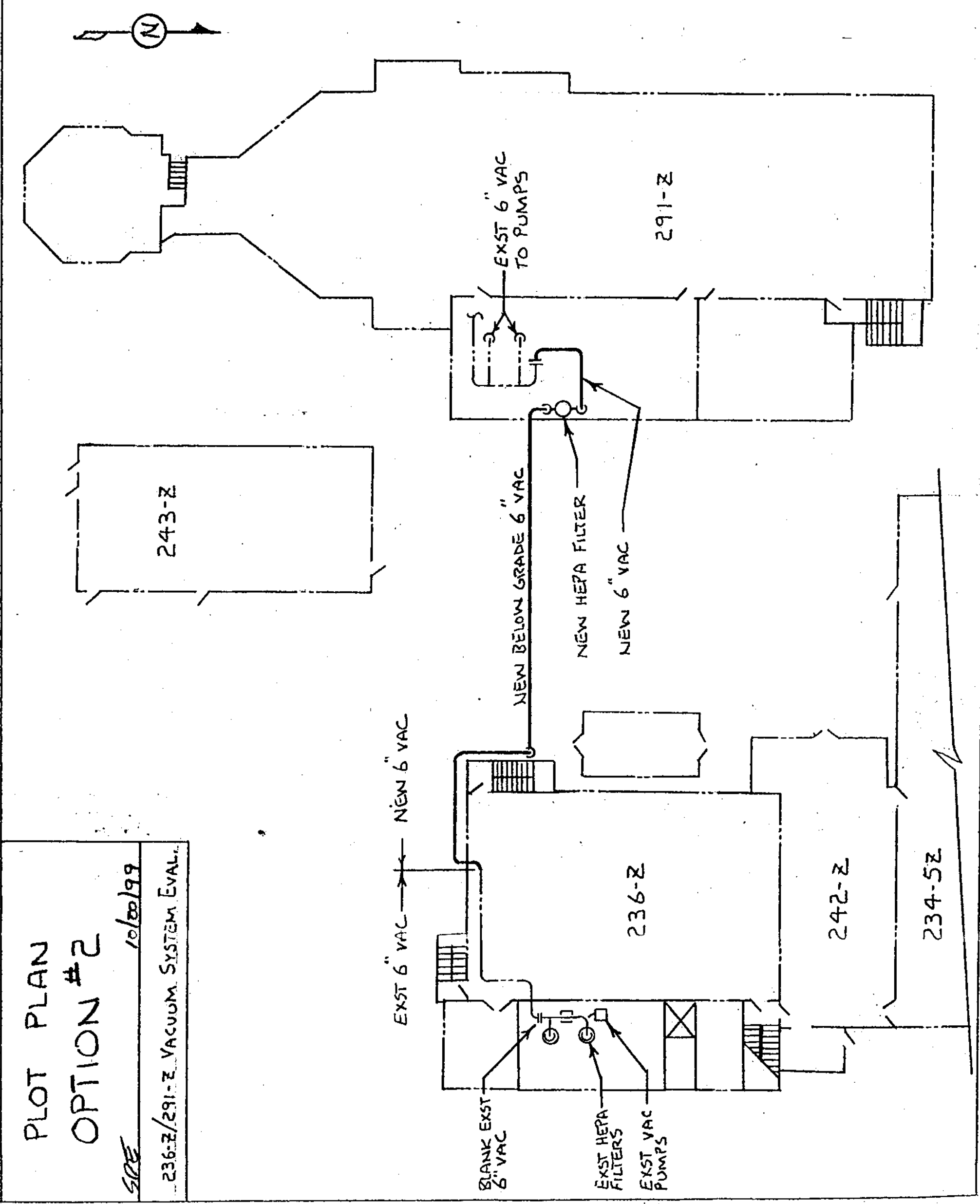




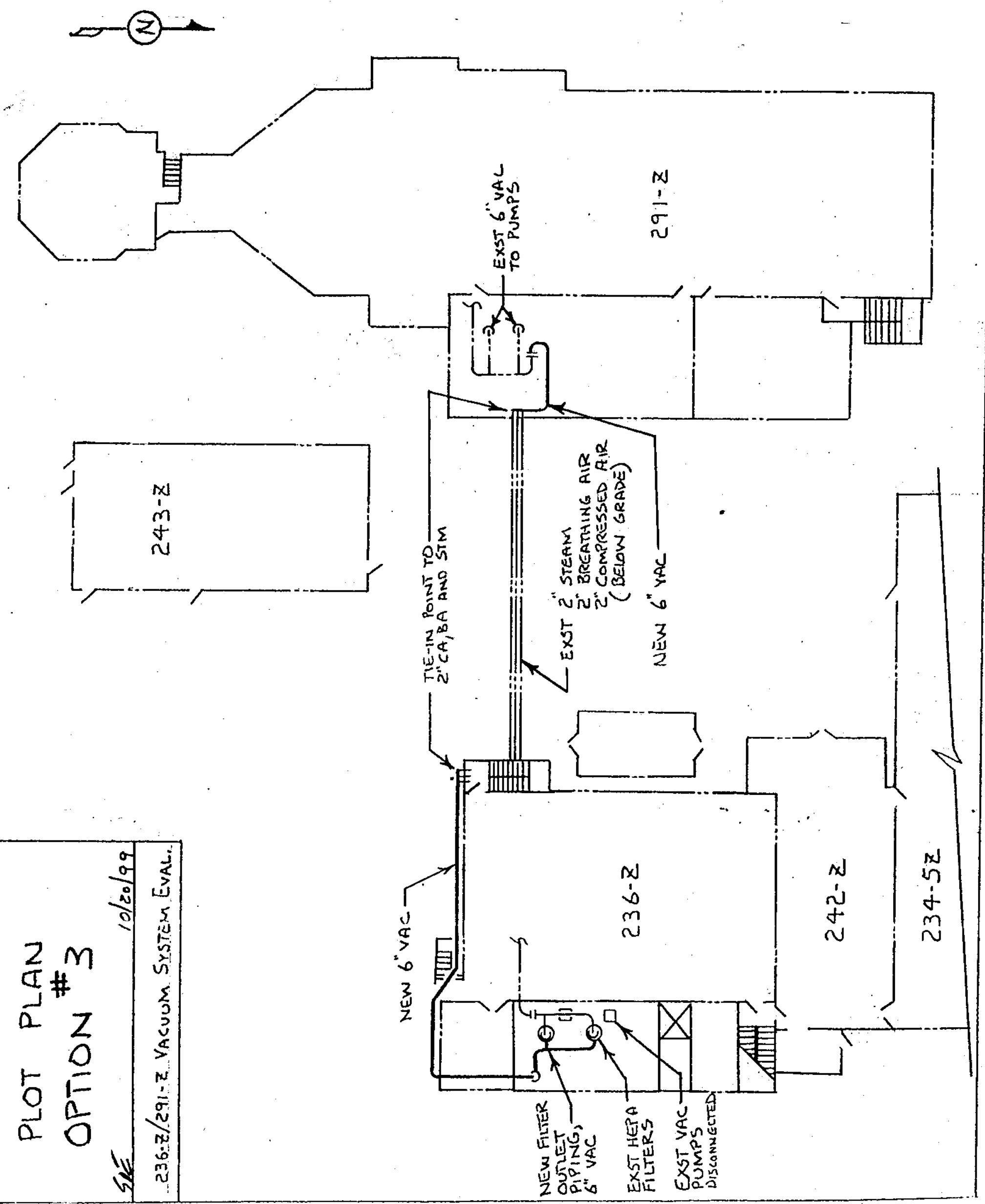




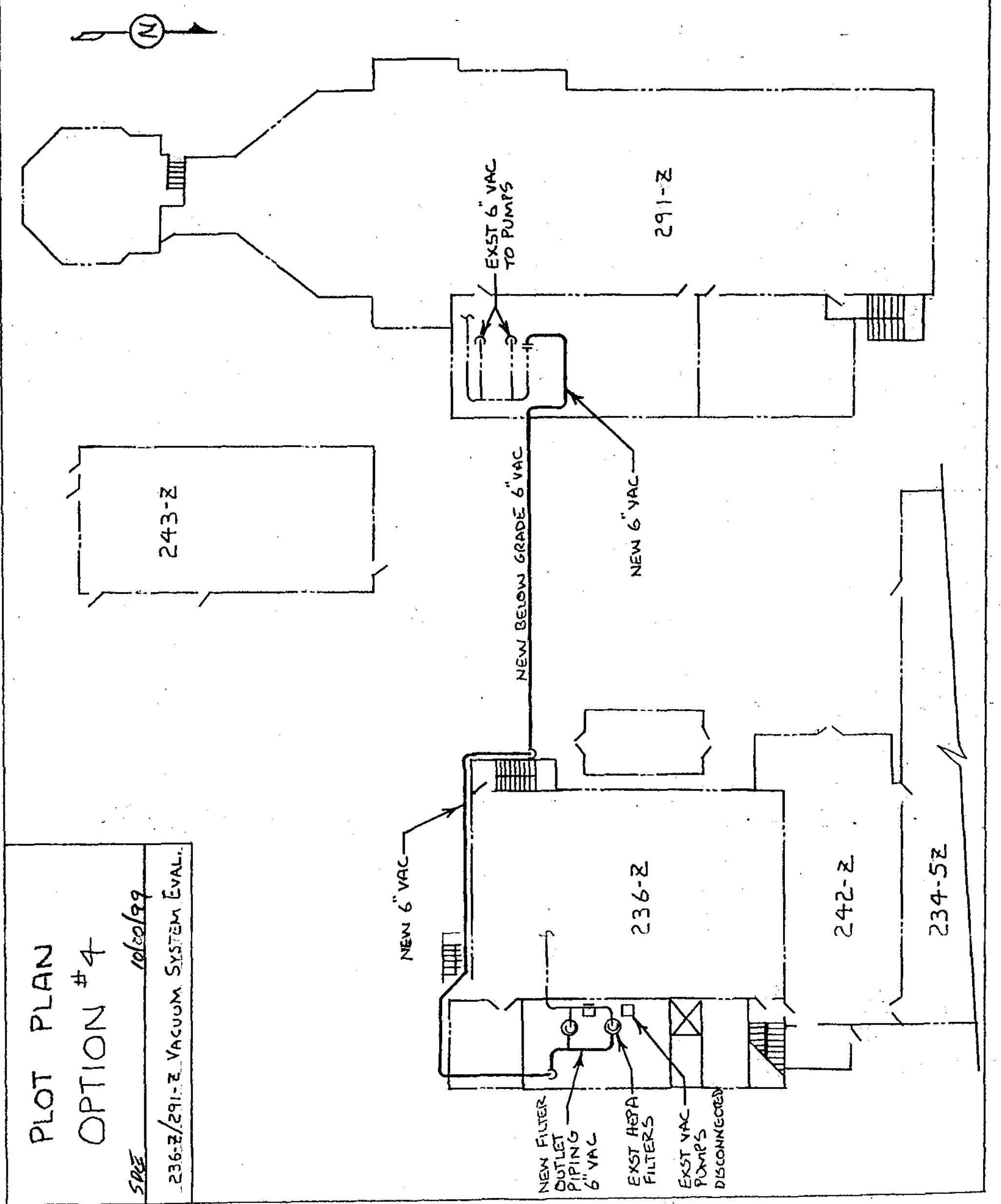

